\title{
Signal Feature Extraction and Combination to Enhance the Detection and Localization of Railroad Track Irregularities
}

\author{
Bhavana Bhardwaj, Raj Bridgelall, Pan Lu, Neeraj Dhingra
}

\begin{abstract}
Tracks are critical and expensive railroad asset, requiring frequent maintenance. The stress from heavy car axle loads increases the risk of deviations from uniform track geometry. Irregularities in track geometry, such as track warping, can cause an excessive harmonic rocking condition that can lead to derailments, traffic delays, and associated financial losses. This paper presents an approach to enhance the location identification accuracy of track geometry irregularities by combining measurements from sensors aboard Hi-Rail vehicles. However, speed variations, position recording errors, low GPS update rates, and the non-uniform sampling rates of inertial sensors pose significant challenges for signal processing, feature extraction, and signal combination. This study introduces a method of extracting features from the fused data of inertial sensors and GPS receivers with multiple traversals to locate and characterize irregularities of track geometry. The proposed method provides robust detection and enhanced accuracy in the localization of irregularities within spatial windows along the track segment. Tradeoff analysis found that the optimal spatial window size is 5 -meter.
\end{abstract}

Index Terms - Sensor, Road Impact Factor, Feature Extraction, Track Geometry, GPS, Inertial Signal.

\section{INTRODUCTION}

$\mathrm{I}^{\mathrm{r}}$ RREGULARITIES in rail geometry is a primary concern of the railroad industry because they can lead to accidents, resulting in traffic delays and financial losses. Repeated stress from heavy axle loads increases the risk of deviations from uniform track geometry [1]. Therefore, railroads spend billions of dollars each year on infrastructure inspections and maintenance [2]. Measures of track geometry include vertical profile, horizontal alignment, cross-level, gage, and warp. The design values of these parameters enable safe and efficient train operation [3].

Regular track geometry monitoring, inspections, and maintenance result in a smoother ride, reduce wear and tear, and ensure safe operations. However, traditional inspection methods are laborious, relatively slow, expensive, and require track closure to search for possible track defects.

B. Bhardwaj, R. Bridgelall, P. Lu, and N. Dhingra are with the Department of Transportation, Logistics, and Finance, North Dakota State University, ND 58108 USA. (Email: bhavana.bhardwaj@ndsu.edu).
Additionally, lack of resources needed and the loss of network capacity limit the ability of railroads to scale inspections for continuous and network-wide monitoring. A promising alternative is to use sensors aboard revenue service trains to identify locations of ride roughness and processing inertial signals to identify the type of track geometry irregularity.

Much of the previous research in this area has explored the concept of using data from onboard sensors to continually monitor the roughness of road pavements [4]-[6]. This method has the potential to reduce the monitoring cost and provide more accurate results. The same technique is applicable to railroads. Dedicated sensors or smartphones can log and transmit time, inertial, and geospatial position data that machine learning algorithms can use to infer track properties. The approach can enhance the efficiency of traditional track inspections by focusing inspection resources on high-risk locations. However, the non-uniform sample rate and inaccurate geospatial position estimates from low-cost GPS receivers pose significant challenges for signal processing, feature extraction, and signal combination [7], [8]. Appropriate noise filtering is necessary to maximize the signal-to-noise ratio (SNR) of each signal prior to feature extraction [9].

This research applies a method of roughness feature extraction called the Road Impact Factor (RIF) transform. The mathematical transform integrates time, inertial signals, and geospatial position data within consecutive distance windows to reduce the data size and produce features called RIF indices. The authors previously demonstrated the use of RIF indices to characterize the intensity of roadway anomalies [5]. Moreover, increases in traversal volume improve the precision of anomaly localization by reducing the variance of the RIF-index. Subsequent experiments found that RIF indices are similarly effective in characterizing ride roughness due to irregular railroad track geometry [10].

The RIF transform uses GPS coordinates to tag the position of each distance window. Therefore, the position of a given distance window reflects the errors and low resolution of GPS position estimates across multiple traversals. The primary contribution of this research is an algorithm that can enhance 
the accuracy of identifying and locating track irregularities by combining the data from multiple traversals of the same or different trains, at various speeds. In particular, the algorithm computes the ensemble average of RIF indices (EAR) across the distance windows of multiple traversals. The EAR reduces noise while enhancing feature detection by leveraging the correlation property of uncorrelated noise and correlated signal, respectively. Equivalently, the EAR enhances the signal-tonoise ratio (SNR), which reduces both false positive and false negative feature detection errors. Furthermore, the analysis determines a distance window size that yields the best tradeoff between the consistency and spatial resolution of estimating the position of a track irregularity.

The organization of this paper is: The literature review section summarizes related work. The data section describes the sensor specifications and placement location. The data section also describes the format and units of the GPS-tagged inertial signal samples. The method section defines and demonstrates the EAR method. The result section evaluates the outcome of the method by comparing the EAR and their corresponding standard deviations as a function of distance window size to determine the best window size for the railroad application. The conclusion section makes concluding remarks about the generalization and utility of the method.

\section{LITERATURE REVIEW}

Several studies have proposed monitoring track networks with sensors aboard in-service vehicles. Lederman et al. [11] presented a data fusion approach for data-driven track monitoring with in-service trains. They mounted two uni-axial accelerometers vertically on two trains and placed a BU-353 GPS receiver under the roof of the first train. Real et al. [12] developed a technique to estimate track profile and relate it to track conditions.

Data fusion is a technique that combines data and information from multiple sensor sources to achieve more specific inferences. A primary advantage of data fusion is improved robustness or a higher fault-tolerance rate. Another advantage is an ability to gain more insights into the situation for improved inference and better decision making. It also improves data accuracy and reduces data uncertainty and ambiguity and extends coverage of information on an observed object or event.

Generally, it is better to use multiple sources of data than to depend on a single source to provide all the necessary information [13], [14]. However, some problems make data fusion a more challenging task. The first problem is sensor selection, which is one of the integration functions that allow a multi-sensor system to select the most appropriate configuration of sensors from among the sensors available. Hence, sensor selection strategies need to be appropriately performed. Another problem is data alignment. The output from different sensors can lead to different dimensionalities and features. Therefore, data alignment and transformation are needed to achieve a common format and standard.

Many researchers related to railroads have used different techniques to access track irregularity. Time and frequency domain analysis methods were effective in identifying track irregularities. Bocciolone et al. [15] developed a system that detects track corrugations by visualizing the wavelet transform of the data acquired from accelerometers mounted on the axle of a passing train. Mori et al. [16] introduced a portable condition monitoring system that estimated irregularities from the vertical and lateral accelerations of a car body. They used a GPS and a map-matching algorithm to identify fault locations on the track. Another study used power spectral density (PSD) to estimate irregularities in track geometry [17]. Zongyi et al. [18] proposed an online monitoring method to detect the vertical long-wavelength track irregularities based on the bogie pitch rate. The method included a mixed-filtering approach for bogie pitch rate and a coupling dynamics model of vertical vehicle-track interactions to obtain simulation data of bogie pitch rate. The result showed that the proposed method could monitor the long-wavelength track irregularities effectively and accurately. Similarly, Zhou et at. [19] developed a web-based conditioning monitoring system that includes a variety of sensors for acquiring trackside data related to different parameters. The system allows a remote operator to download data automatically for offline analysis while observing the health condition of the point machine using Internet Explorer.

Another monitoring application [20] that used a light source and high-resolution cameras found an increase in false positives due to stains from oil and dust particles. Santur, Karaköse, and Akın used 3D laser cameras to provide high accuracy rates in real-time and found that the system was prone to false positives [21]. The cost of these applications is high because of the need for high accuracy of detection at high speeds [21].

Some authors [22], [23] focused on developing approaches that aggregate and evaluate track geometry measures across varying lengths of track. Those studies used the standard deviation of track geometry as an index to represent track surface roughness. The track geometry condition indicator obtained through standard deviation is called the Track Quality Index (TQI) [23]. Sadeghi [24] developed indexes of rail track geometry based on the statistical distribution of track geometry data. They found that the field data obtained from four geometrical parameters, namely gauge, profile, alignment, and twist, follow a normal distribution.

All these studies demonstrated a successful assessment of irregular track geometry algorithms. However, they all focused on the use of complex and expensive sensors and cameras, which result in high maintenance costs. Moreover, the life cycle of such sensors is limited due to the high-impact forces of wheel-rail interaction [25]. Therefore, a research gap is characterizing the effects of combining the signals from 
multiple train traversals at different speeds to detect track irregularities throughout the network.

\section{METHOD}

This section describes the methods used to enhance the detectability and location estimation accuracy of track irregularities. The authors selected the Road Impact Factor (RIF) space-time transform to extract inertial features within consecutive distance windows along the traversal path [5]. This method has the advantages of simultaneous data reduction and feature extraction, at any speed. Prior to producing the EAR, the workflow included distance interpolation from a spatial reference position to align the signals and to extract approximately equal length segments, as described in previous work [26]. Additional processes described in previous work included computing a Fast Fourier Transform (FFT) and ensemble average of the FFT (EA-FFT) across multiple signals to inform an appropriate filter cutoff frequency, and applying a finite impulse response (FIR) low-pass filter [9]. Fig. 2 illustrates the complete workflow.

Irregular track geometry produces at least one maxima in the inertial signal [5]. Fig. 3 compares the inertial signals from two random traversals of an isolated bump. The second signal is at an offset for clarity. The bump produced a first major valley (FMV) as illustrated. The distance is relative to a reference position near the beginning of each traversal. The algorithm interpolates the distance from that position by using the speed and time samples.

\section{A. Feature Extraction}

The RIF transform reduces the data into features that are proportional to the roughness within a distance window $\Delta \mathrm{L}$ such that

$R^{\Delta L}=\sqrt{\frac{1}{\Delta L} \sum_{n=0}^{N-1}\left|G_{\mathrm{z}[n]} v_{n}\right|^{2} \delta t_{n}}$

where the RIF index $R^{\Delta L}$ is the average $g$-force magnitude per unit of distance $\Delta \mathrm{L}$ travelled. $G_{\mathrm{z}[\mathrm{n}]}$ is the vertical acceleration for signal sample $\mathrm{n}$. The sample period instant is $\delta t_{n}$ and, the instantaneous traversal speed is $v_{n}$.

\section{B. Ensemble Average of RIF Indices (EAR)}

The window position varied with GPS error. The RIF intensity varied with traversal speed. Hence, an ensemble average of the RIF-indices (EAR) within a selected distance window along the traversal path and across $\mathrm{N}$ traversals produced an estimate of the average roughness $E^{w}$ experienced in window $\mathrm{w}$, at any speed where

$E^{w}=\frac{1}{N} \sum_{x=1}^{n} R_{x}^{w}$.
$R_{x}^{w}$ is the RIF index from traversal $\mathrm{x}$ within distance window $\mathrm{w}$. The intensity of the peak EAR is proportional to the amount of roughness produced from traversing roadway or railroad track anomalies [5]. Fig. 4a and fig. 4b shows the EAR of the road segment data for spatial resolution windows of $1,5,15$, and 20 meters. The chart shows the unprocessed inertial signal from one of the traversals for reference. The position of the maximum EAR is an estimate of the position of the track irregularity. The precision of position estimate increases with the number of traversals. Larger window sizes provide greater data reduction but reduce the precision of estimating the position of an irregularity.

\section{Ensemble STD RIF}

The ensemble STD RIF (ESR) is

$\sigma_{R I F}=\sqrt{\frac{\sum_{x=1}^{N}\left(R_{x}^{W}-E^{w}\right)^{2}}{N}}$

It is a measure of the variability of the roughness intensity across traversals, within a distance window. Fig. $4 \mathrm{c}$ and fig. $4 \mathrm{~d}$ show the ESR of the traversal data with distance windows of 1, 5, 15, and 20 meters. The ESR generally declines as the window size increases because the EAR also decreases. This represents a tradeoff in data size for accuracy and precision in estimating the position of anomalies.

\section{Margin-of-Error}

The margin-of-error (MOE) is calculated with a $95 \%$ confidence interval (CI) as a function of number of traversals within the selected window sizes such that

$$
\mathrm{MOE}=Z * \frac{\sigma}{\sqrt{n}}
$$

where $\mathrm{z}$ is the critical value of 1.96 and $\sigma$ is the standard deviation of the intensity of the peak RIF within the selected distance window sizes. The total number of traversals within the window size is n. Fig. 6 shows the MOE of the peak RIF for distance windows of 1, 2, 5, 10, 15, 20 and 40 meters. The chart shows that the MOE declines most substantially between window sizes of 1 and 5 meters. This suggests that that the reliability of the estimate is best for the window sizes in that range.

\section{EXPERIMENTAL SETUP}

\section{A. Data Collection Site \& Sensor Specification}

Smartphones provide a flexible and convenient tool to develop and test the viability of an automated track monitoring system that uses dedicated sensors onboard regular service trains [27], [28]. In previous work, the authors verified that the inertial signal patterns observed from traversing irregular track geometry are similar to those observed when a sedan traverses pavement anomalies [9]. The study concluded that the method 
applies identically on actual railroad detect and localize railroad track anomalies.

For the first experiment, the authors used a sedan to emulate train traversals across irregular track geometry. A smartphone app named pavement analysis via vehicle electronic telemetry (PAVVET) logged the inertial and geospatial data from a sedan by driving a paved road segment (Cell 40) of the Minnesota road (MnROAD) research facility in the United States. MnROAD is an outdoor laboratory that supports the performance testing of various pavement types [29]. The smartphone was mounted flat onto the dashboard of the sedan with the y-axis pointing in the direction of travel [26].

The PAVVET iOS $®$ app utilized all the required sensors on a smartphone [30]. The embedded sensors are an STMicroelectronics LIS331DLH accelerometer, a L3G4200D gyroscope, and a global positioning system receiver [31], [32]. The smartphone (iPhone 4S®) sampled the accelerometer at a nominal rate of 128 hertz. The GPS receiver sampled at a nominal rate of one hertz. Additionally, it is well known that the geospatial position estimates from low-cost GPS receivers are inaccurate, mostly because of signal deterioration in urban environments and their low update rate. Thus, the author [27] presented a method to estimate the position of anomalies with greater accuracy when using low-cost GPS receivers onboard regular vehicles. The estimated position is a simple function of the average traversal speed, the GPS update interval, and the system latency in tagging accelerometer samples with GPS coordinates. The experiment result shows that with a good estimate of the system latency, the method provides sub-meter accuracy. Moreover, iPhone 4 determined approximately $98 \%$ of its GPS points within $10 \mathrm{~m}$ of true positions and approximately $59 \%$ within $5 \mathrm{~m}$ [33].

The authors conducted a second experiment on a local railroad by placing the same sensors on a hi-rail vehicle. The collect data was from the traversal of a rail grade crossing (RGC) because it reliably simulated the presence of a rail anomaly.

\section{B. Data Format}

Table 1 shows a fragment of the data and its format. The time column is the sample period in milliseconds. The inertial signals "Gx," "Gy," and "Gz" are linear accelerations in the lateral, longitudinal, and vertical directions, respectively, with values in units of g-force. The ground speed (GSpeed) is in units of meters-per-second (m s-1). The GPS receiver produced latitude (Lat) and longitude (Lon) in decimal format, and the RotX, RotY, and RotZ signals are the angular rotations in degrees-per-second around the $\mathrm{X}, \mathrm{Y}$, and Z-axis, respectively. Pitch, roll, and yaw are gyroscope angles in three dimensions that are instantaneous samples in units of degree. The dataset contains a consistent inertial event from a narrow bump at the beginning of each traversal. The distinct inertial signal from this bump produced a first major valley (FMV) in the accelerometer signal. This signal is identical to the inertial signal produced when a railroad car traversed a verified track irregularity near a rail-grade crossing. The isolated irregularity of the dataset served as a fixed position ground truth for position error evaluation. The non-uniform sampling of the accelerometer produced a non-linear sample period among traversals. Fig. 1 shows the distribution of the sample period collected across 53 traversals of the road segment. From the chart, the sample period is ranging from 1 to 18 milliseconds. However, it is also clear that the top bar is approximately 11 milliseconds, which is equal to a sample rate of 90 hertz. Moreover, the author [31] found that the inertial sample rate must be at least 64 hertz for the reliable detection of roadway anomalies.

The non-linear sampling decreases signal strength and increases noise. The authors also found that it is practically impossible to force the smartphone to sample uniformly at a specific rate, despite the settings for a sample rate. However, setting it at the maximum rate, and subsequently applying a low-pass filter normalized the effect of sampling at different rates because energy was removed after the same frequency for the data from each traversal [9].

Consequently, pre-processing with an appropriate low-pass filter maximized the SNR for subsequent feature extraction [9]. The pre-processing includes ensemble averaging of the inertial signals from the same position along the traversal path. Therefore, the approach will increase the quality of the composite signal with each additional data stream combined. Basically, averaging reduces noise in the composite signal because noise is uncorrelated [34]. Moreover, the authors [27] demonstrated that the smartphones running the Android operating system and iOS operating system sampled at their highest rate. The iOS models sampled between 91 and 134 hertz, whereas the Android sampled at approximately 385 hertz. The mean sample rate of the Android smartphones differed by approximately 1 hertz due to the statistical nature of the sampling, as described in [34].

\section{RESULT \& DISCUSSION}

Before producing the EAR and ESR, the algorithm interpolated the distance from a reference position near the beginning of each traversal by using speed and time samples [34], and applied the RIF transform to extract inertial features within consecutive distance windows along the traversal path. From the road experiment, Fig. $4 \mathrm{a}$ and $4 \mathrm{~b}$ show that the position of the maximum EAR is an estimate of the position of the irregularity. Fig. 4c and 4d show that the ESR declines as the window size increases, which is the expected outcome. As a function of window size, Table 2 summarizes the means and standard deviations (STD) of the peak RIF intensity and the center position of the window for the peak RIF relative to the 
beginning of the traversals. A smaller window size increases the precision of locating an irregularity but also increases the variability of that estimate. The results show that an optimum window size exists that minimizes the STD of estimating the RIF intensity. A lower STD improves the consistency of detecting an anomaly and, therefore, reduces the false positive and false negative detection errors. Fig. 5a shows the mean and standard deviation of the intensity of the peak RIF within the selected distance window sizes. Fig. 5b indicates that keeping the distance window size below 5 meters maintains a stable estimate of the position of the peak RIF relative to the beginning of the traversal (approximately 53 meters for the experiment), and minimizes the STD of that estimate.

Additionally, table 3 shows the margin-of-error (MOE) of peak RIF within the selected window size. Fig. 6 indicates that MOE provides the confidence of the measurements with an indication that there is an optimum window size selection.

From the rail experiment, Table 4 represents the mean and STD of the peak RIF intensity relative from the beginning of each traversal. Fig. 7a shows that the RIF transform presents a stable trade-off between resolution and confidence as a function of window size with an asymptotic decrease of the mean value and a consistent decrease in the STD of the estimate. Fig. 7b reveals that a window size of 5 meters provides a stable estimate for locating rail anomalies relative to a linear reference at the beginning of the traversals, which was approximately 90 meters in these experiments.

A window size of 5 meters in both the road and rail experiments balances the tradeoff in data size, precision, and accuracy of locating road or rail irregularities with sensors. The best window size is also within the visual range for inspectors to locate the anomaly.

\section{CONCLUSION}

A reliable, low-cost method for detecting and identifying the location of irregular rail track geometry can enhance the efficiency of traditional track inspections by focusing inspection resources on high-risk locations. The use of sensors aboard regular service trains enhances coverage and monitoring frequency. However, GPS and sensor errors diminish the attainable accuracy and precision of detecting the presence and location of an irregularity.

This paper introduces an analytic framework that includes mathematical and statistical methods to enhance the detection and localization accuracy of track irregularities by extracting and combining features of the inertial signals obtained from multiple traversals of a track segment. The method employs the Road Impact Factor (RIF) space-time transform, which provides the advantage of data reduction and feature extraction within adjustable and consecutive distance windows along the traversal path. The ensemble average of RIF-indices within each window provides an estimate for the intensity and position of an irregularity. Increasing the window size decreases the variability of the intensity estimate and decreases the data size. However, a larger spatial window size also reduces the accuracy and precision of position estimates. Both roadway and railway experiments were conducted to demonstrate the reliability and accuracy of the employed method. In both experiments, tradeoff analysis found that a window size of 5 meters provided a good balance between data reduction, accuracy, precision, and the consistency of anomaly detection while minimizing the potential for false positives and false negatives. A significant benefit of the ensemble averaging approach is that both precision and accuracy increase with the number of traversals.

Furthermore, the MOE of peak RIF values also validates confidence in the measurements and points to an optimal window size selection.

Future work will focus on classifying and quantifying irregularity types including profile, alignment, and warp by using the proposed analytical framework.

\section{ACKNOWLEDGMENT}

The work presented in this paper was conducted with support from North Dakota State University and the Mountain-Plains Consortium, a University Transportation Center funded by the U.S. Department of Transportation. The contents of this paper reflect the views of the authors, who are responsible for the facts and accuracy of the information presented.

\section{REFERENCES}

FRA, "Track inspector rail defect reference manual," Off. Railr. Safety, US Dep. Transp., vol. 2, no. July, 2015.

[2] USDA, "Grain Transportation Report," Agricultural Marketing Service, 2018. [Online]. Available: www.ams.usda.gov/GTR. [Accessed: 14-Dec-2019].

[3] M. Fateh, "Fractal Analysis of Geometry Data for Railroad Track Condition Assessment," Fed. Railr. Adm., no. December, pp. 1-4, 2000.

[4] G. W. Flintsch, B. Ferne, B. Diefenderfer, S. Katicha, J. Bryce, and S. Nell, "Evaluation of Traffic-Speed Deflectometers," Transp. Res. Rec. J. Transp. Res. Board, vol. 2304, no. 1, pp. 37-46, 2012.

[5] R. Bridgelall, "Connected Vehicle Approach for Pavement Roughness Evaluation,” J. Infrastruct. Syst., vol. 20, no. 1, p. 04013001, 2014.

[6] A. Malekjafarian, E. J. Obrien, and D. Cantero, "Railway Track Monitoring Using Drive-By Measurements," in The Fifteenth East Asia-Pacific Conference on Structural Engineering and Construction (EASEC-15), 2017, pp. 1-7.

[7] N. A. Ali and M. Abu-Elkheir, "Improving localization accuracy: Successive measurements error modeling," Sensors, vol. 15, no. 7, pp. 15540-15561, 2015.

[8] R. Schenkendorf and B. Dutschk, "Improved Railway Track Irregularities Classification by a Model Inversion Approach," in In Proceedings of the Third European Conference of the Prognostics and Health Management Society 2016, Bilbao, Spain, 2016, pp. 358365.

[9] B. Bhardwaj, R. Bridgelall, L. Chia, P. Lu, and N. Dhingra, "Signal Filter Cut-off Frequency Determination to Enhance the Accuracy of Rail Track Irregularity Detection and Localization,” IEEE Sens. J., vol. 20, no. 3, pp. 1-1, 2019. 
[10] B. Bhardwaj, R. Bridgelall, P. Lu, and N. Dhingra, "Railroad Track Irregularities Position Accuracy Assessments using Low-Cost Sensors on a Hi-Rail Vehicle," in International Conference on Transportation and Development 2019, 2019, pp. 458-465.

[11] G. Lederman, S. Chen, J. H. Garrett, J. Kovačević, Y. H. Noh, and J. Bielak, "A data fusion approach for track monitoring from multiple in-service trains," Mech. Syst. Signal Process., vol. 95, pp. 363-379, 2017.

[12] J. Real, P. Salvador, L. Montalbán, and M. Bueno, "Determination of rail vertical profile through inertial methods," Proc. Inst. Mech. Eng. Part F J. Rail Rapid Transit, vol. 225, no. 1, pp. 14-23, 2011.

[13] B. Khaleghi, A. Khamis, F. O. Karray, and S. N. Razavi, "Multisensor data fusion: A review of the state-of-the-art," Inf. Fusion, vol. 14, no. 1, pp. 28-44, 2013.

[14] G. W. Ng, Intelligent System Fusion, Tracking and Control. Research Studies Press Ltd., 2003.

[15] M. Bocciolone, A. Caprioli, A. Cigada, and A. Collina, "A measurement system for quick rail inspection and effective track maintenance strategy," Mech. Syst. Signal Process., vol. 21, no. 3, pp. 1242-1254, 2007.

[16] H. Mori, H. Tsunashima, T. Kojima, A. Matsumoto, and T. Mizuma, "Condition Monitoring of Railway Track Using In-service Vehicle," J. Mech. Syst. Transp. Logist., vol. 3, no. 1, pp. 154-165, 2010.

[17] R. N. Iyengar and O. R. Jaiswal, "Closure to 'Random Field Modeling of Railway Track Irregularities' by R. N. Iyengar and O. R. Jaiswal," J. Transp. Eng., vol. 121, no. 4, pp. 303-308, 1995.

[18] X. Zongyi, C. Yuejian, and Q. Yong, "Online monitoring of vertical long wavelength track irregularities using bogie pitch rate," $J$. Vibroengineering, vol. 17, no. February, pp. 216-228, 2015.

[19] F. B. Zhou, M. D. Duta, M. P. Henry, S. Baker, and C. Burton, "Remote condition monitoring for railway point machine," in ASME/IEEE 2002 Joint Rail Conference, RTD 2002, 2002, pp. 103108.

[20] C. Alippi, E. Casagrande, F. Scotti, and V. Piuri, "Composite realtime image processing for railways track profile measurement," IEEE Trans. Instrum. Meas., vol. 49, no. 3, pp. 559-564, 2000.

[21] E. Santur, Yunus; Karaköse,Mehmet; Akın, "Learning Based Experimental Approach For Condition Monitoring Using laser cameras in railway tracks," Int. J. Appl. Math. Electromics Comput., pp. 1-5, 2016.

[22] M. El-Sibaie and Y.-J. Zhang, "Objective Track Quality Indices," Transp. Res. Rec. J. Transp. Res. Board, vol. 1863, no. 1, pp. 81-87, 2007.

[23] P. Xu, R. Liu, Q. Sun, R. R. Souleyrette, and J. G. Rose, "A Novel and Reliable Track Condition Prediction Model for Condition Based Track Maintenance," in Proceeding of the 2013 Joint Rail Conference, 2013, no. April, p. V001T01A005-V001T01A005.

[24] J. Sadeghi, "Development of Railway Track Geometry Indexes Based on Statistical Distribution of Geometry Data," J. Transp. Eng., vol. 136, no. 8, pp. 693-700, 2010.

[25] L. Chia, B. Bhardwaj, P. Lu, R. Bridgelall, and S. Member, "Railroad Track Condition Monitoring Using Inertial Sensors and Digital Signal Processing : A Review," vol. 19, no. 1, pp. 25-33, 2019.

[26] L. Chia, B. Bhardwaj, R. Bridgelall, P. Lu, D. Tolliver, and N. Dhingra, "Transportation Research Record Heuristic Methods of Inertial Signal Alignment to Detect and Locate Railtrack," in Presented at the 98th Annual Meeting of the Transportation Research, Washington, DC, USA, 2019.

[27] R. Bridgelall and D. Tolliver, "Accuracy enhancement of anomaly localization with participatory sensing vehicles," Sensors, vol. 20, no. 2, pp. 1-15, 2020.

[28] N. D. Lane, E. Miluzzo, H. Lu, D. Peebles, T. Choudhury, and A. T. Campbell, "A survey of mobile phone sensing," IEEE Communications Magazine, vol. 48, no. 9, pp. 140-150, 2010.

[29] R. Bridgelall, M. T. Rahman, J. F. Daleiden, and D. Tolliver, "Error sensitivity of the connected vehicle approach to pavement performance evaluations," Int. J. Pavement Eng., vol. 19, no. 1, pp. 82-87, 2018.

[30] R. Bridgelall, Y. Huang, Z. Zhang, and F. Deng, "Precision enhancement of pavement roughness localization with connected vehicles," Meas. Sci. Technol., vol. 27, no. 2, 2016.

[31] R. Bridgelall, "Inertial Sensor Sample Rate Selection for Ride Quality Measures," J. Infrastruct. Syst. ASCE, vol. 21, no. 2, p. 04014039, 2014.

[32] A. Allan, "Using the Accelerometer," in Basic Sensors in iOS, 1st ed. CA, USA: O'Reilly Media, Inc., 2011.

[33] T. Menard, J. Miller, M. Nowak, and D. Norris, "Comparing the GPS capabilities of the Samsung Galaxy S, Motorola Droid X, and the Apple iPhone for vehicle tracking using FreeSim-Mobile," IEEE Conf. Intell. Transp. Syst. Proceedings, ITSC, pp. 985-990, 2011.

[34] R. Bridgelall, L. A. Chia, B. Bhardwaj, P. Lu, D. D. Tolliver, and N. Dhingra, "Enhancement of signals from connected vehicles to detect roadway and railway anomalies," Meas. Sci. Technol., vol. 31, no. 3, pp. 1-11, 2019.

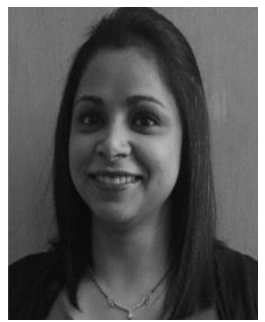

Bhavana Bhardwaj is working toward her Ph.D. degree within the Department of Computer Science and is a research assistant in the Department of Transportation, Logistic, and Finance at North Dakota State University. Her research interests include transportation safety, data mining techniques in transportation, sensor application in transportation and their application to signal processing.

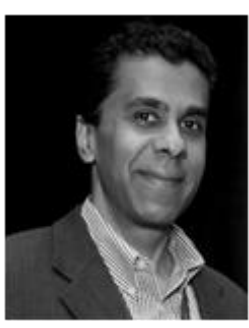

Dr. Raj Bridgelall (M'91-SM'02) Bridgelall received a Ph.D. degree in transportation and logistics at the North Dakota State University (NDSU), Fargo in 2015. He is a program director of the SMARTSe $^{\mathrm{SM}}$ Intelligent Transportation Systems Center, and an assistant professor with the Department of Transportation, Logistics, and Finance, NDSU.

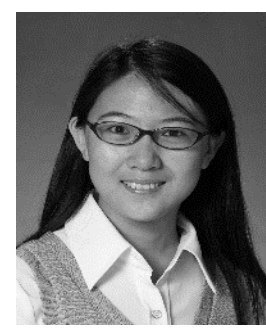

Dr. Pan Lu became a member of IEEE in 2017. She received a Ph.D. degree in transportation and logistics from North Dakota State University in 2011. Since 2017, Dr. Lu has been an associate professor in the College of Business at NDSU. 


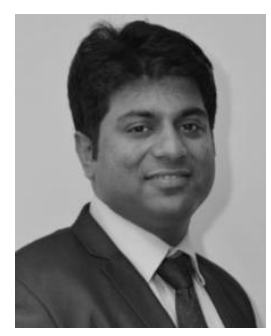

Neeraj Dhingra is currently working toward a Ph.D. degree within the Department of Transportation, Logistics, and Finance at North Dakota State University. His research interests include data mining techniques in transportation, transportation safety, GIS application in transportation and railway safety, and project management.

TABLE I

FORMAT OF DATA SAMPLE

\begin{tabular}{ccccccccccccc} 
Time & Gz & Lat & Lon & GSpeed & Pitch & Roll & Yaw & Gx & Gy & RotX & RotY & RotZ \\
\hline 44.142 & -1.057 & 45.263 & -93.71 & 9.586 & 6.693 & 4.886 & -0.319 & -0.088 & -0.152 & 3.177 & 0.629 & -0.452 \\
46.768 & -1.216 & 45.263 & -93.71 & 9.586 & 6.693 & 4.886 & -0.319 & 0.047 & -0.241 & 3.177 & 0.629 & -0.452 \\
50.26 & -1.087 & 45.263 & -93.71 & 9.586 & 6.693 & 4.886 & -0.319 & 0.026 & -0.272 & 3.177 & 0.629 & -0.452 \\
62.927 & -0.854 & 45.263 & -93.71 & 9.586 & 6.741 & 4.903 & -0.329 & -0.002 & -0.212 & 1.246 & -0.13 & -0.332 \\
73.909 & -0.912 & 45.263 & -93.71 & 9.586 & 6.752 & 4.907 & -0.332 & 0.022 & -0.161 & 1.865 & 0.214 & -0.258 \\
86.754 & -0.942 & 45.263 & -93.71 & 9.586 & 6.776 & 4.908 & -0.341 & 0.038 & -0.144 & 2.005 & -0.67 & -0.189 \\
\hline
\end{tabular}

TABLE II

STATISTICAL PARAMETERS OF PEAK RIF WITHIN SELECTED RESOLUTION WINDOW.

\begin{tabular}{ccccc} 
Window Size & $\begin{array}{c}\text { Peak RIF } \\
\text { Mean }\end{array}$ & Peak RIF STD & $\begin{array}{c}\text { Peak RIF Dist } \\
\text { Mean }\end{array}$ & $\begin{array}{c}\text { Peak RIF Dist } \\
\text { STD }\end{array}$ \\
\hline 1 & 1.48 & 0.60 & 53.38 & 4.02 \\
2 & 1.21 & 0.57 & 53.59 & 4.02 \\
5 & 0.87 & 0.56 & 54.47 & 8.50 \\
10 & 0.70 & 0.56 & 56.79 & 15.91 \\
15 & 0.60 & 0.56 & 56.15 & 20.15 \\
20 & 0.53 & 0.57 & 51.98 & 5.91 \\
40 & 0.43 & 0.58 & 101.22 & 56.57 \\
\hline
\end{tabular}




\begin{tabular}{ccc}
$\begin{array}{c}\text { Window } \\
\text { Size }\end{array}$ & $\begin{array}{c}\text { No. of } \\
\text { Traversals }\end{array}$ & $\begin{array}{c}\text { MOE Peak } \\
\text { RIF }\end{array}$ \\
\hline 1 & 53 & 0.162 \\
2 & 53 & 0.153 \\
5 & 53 & 0.151 \\
10 & 53 & 0.151 \\
15 & 53 & 0.151 \\
20 & 53 & 0.153 \\
40 & 53 & 0.156 \\
\hline
\end{tabular}

TABLE IV

STATISTICAL PARAMETERS OF PEAK RIF WITHIN SELECTED RESOLUTION WINDOW USING RAIL DATA.

\begin{tabular}{ccccc} 
Window Size & $\begin{array}{l}\text { Peak RIF } \\
\text { Mean }\end{array}$ & $\begin{array}{l}\text { Peak RIF } \\
\text { STD }\end{array}$ & $\begin{array}{c}\text { Peak RIF Dist } \\
\text { Mean }\end{array}$ & $\begin{array}{c}\text { Peak RIF Dist } \\
\text { STD }\end{array}$ \\
\hline 1 & 0.764 & 0.187 & 90.114 & 43.780 \\
2 & 0.635 & 0.145 & 86.713 & 30.112 \\
5 & 0.498 & 0.097 & 87.263 & 29.889 \\
10 & 0.424 & 0.074 & 91.513 & 21.590 \\
15 & 0.387 & 0.063 & 88.512 & 28.958 \\
20 & 0.370 & 0.054 & 86.013 & 27.988 \\
40 & 0.335 & 0.059 & 98.013 & 8.945 \\
\hline
\end{tabular}

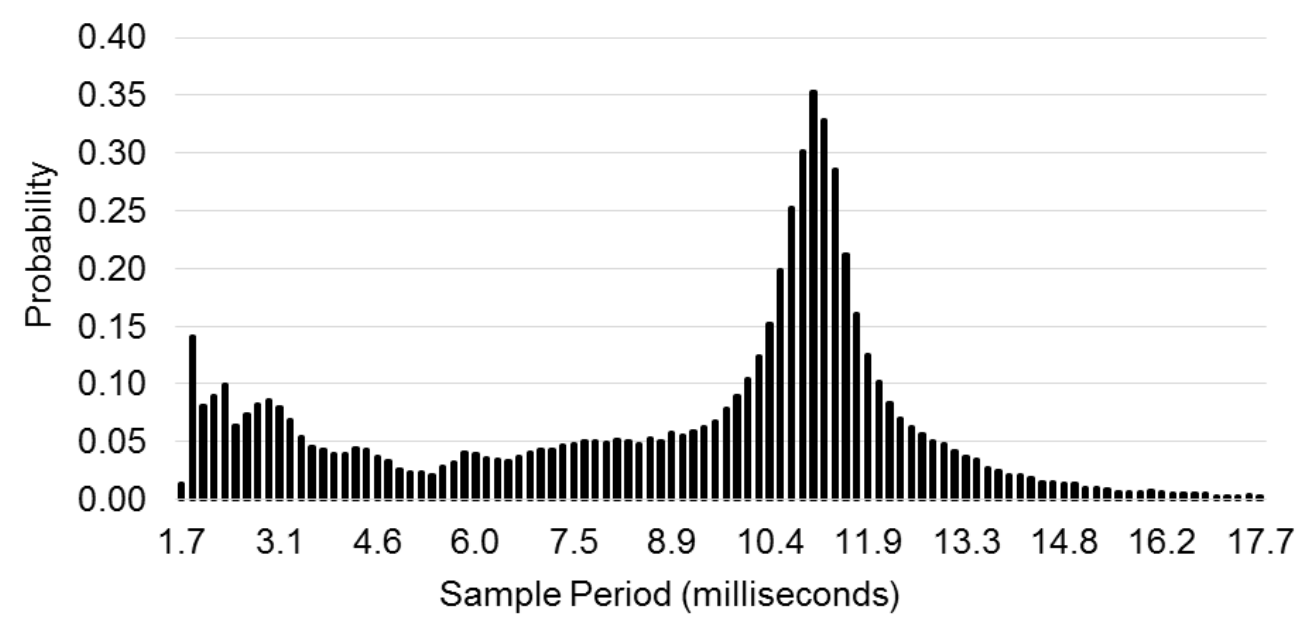

Fig. 1. Distribution of sample periods from accelerometer data across all traversals. 


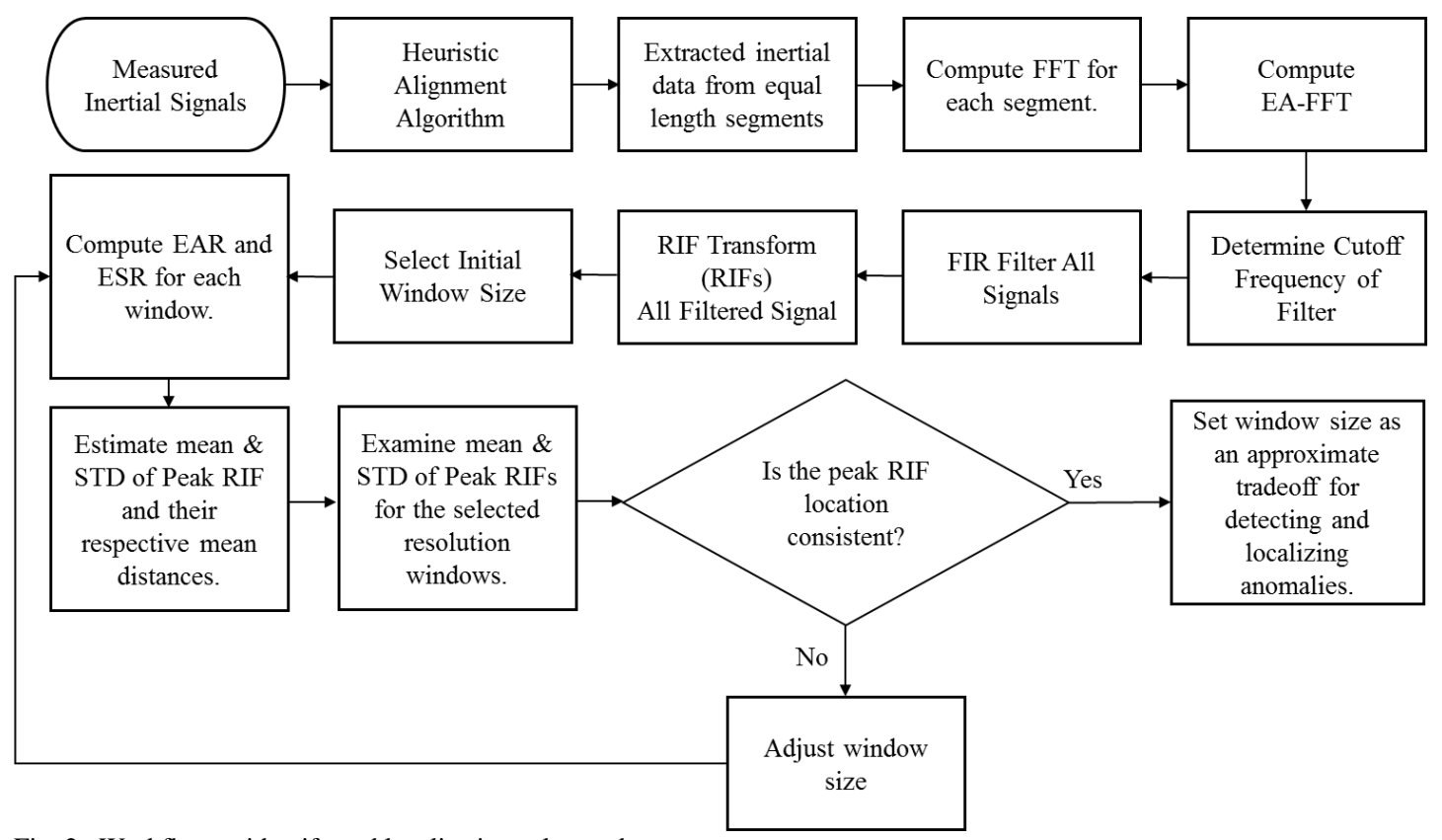

Fig. 2. Workflow to identify and localize irregular track geometry.

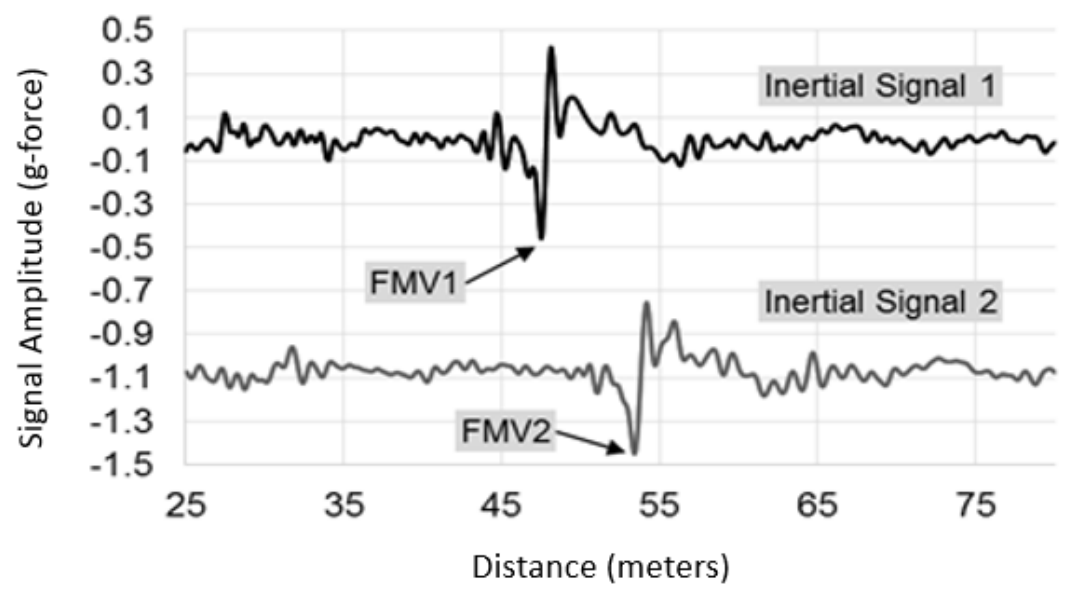

Fig. 3. Position variation of the FMV among two signal streams. 
a)

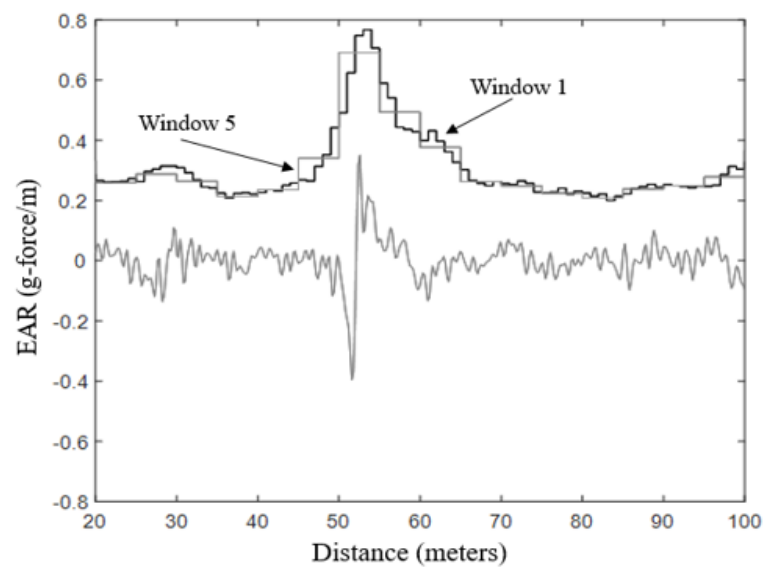

c)

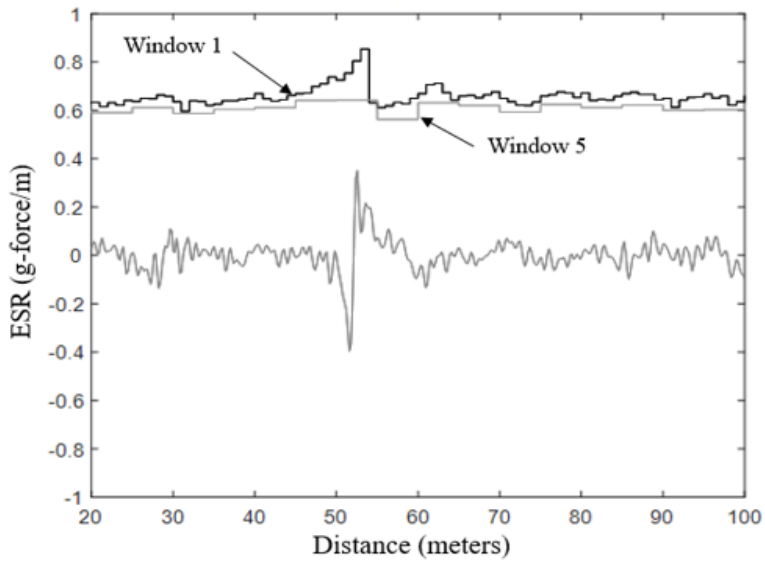

b)

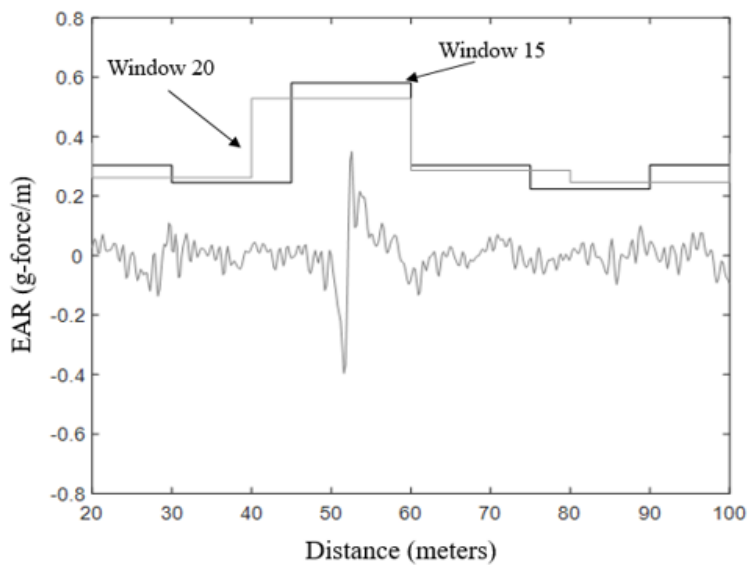

d)

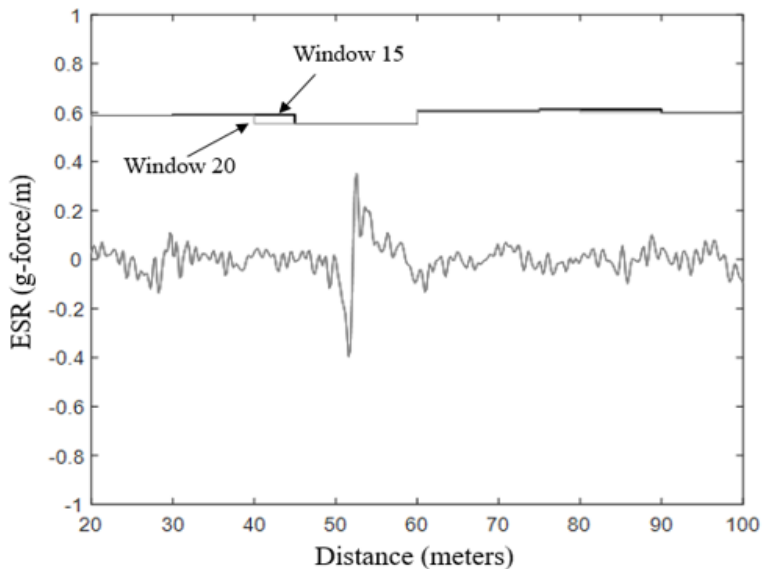

Fig. 4. a) EAR at resolution window of 1 and 5 meters b) EAR at resolution window of 15 and 20 meters c) ESR at resolution window of 1 and 5 meters d) ESR at resolution window of 15 and 20 meters. 
a)

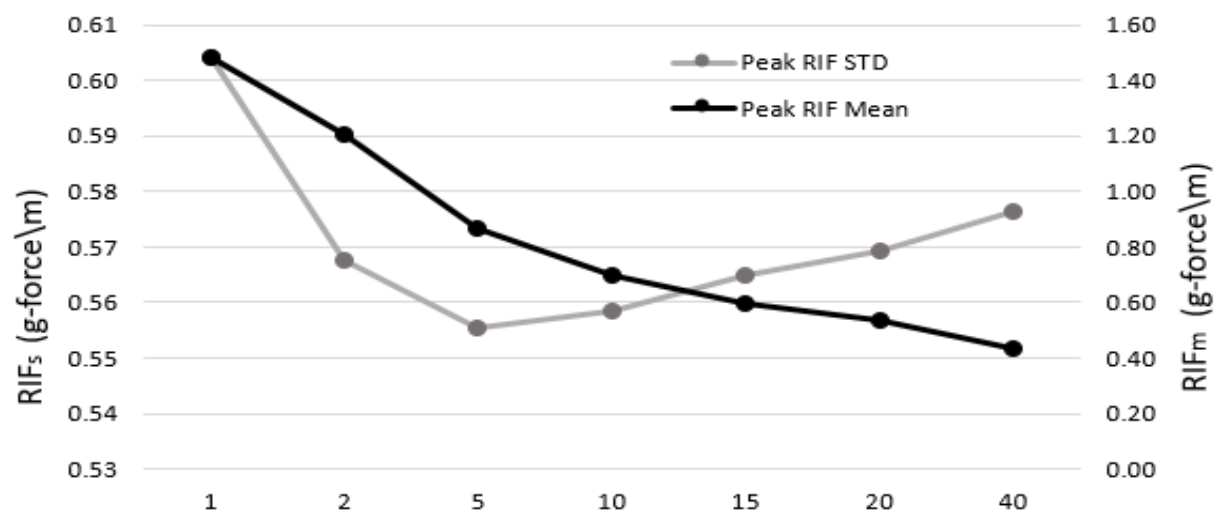

Window size (meters)

b)

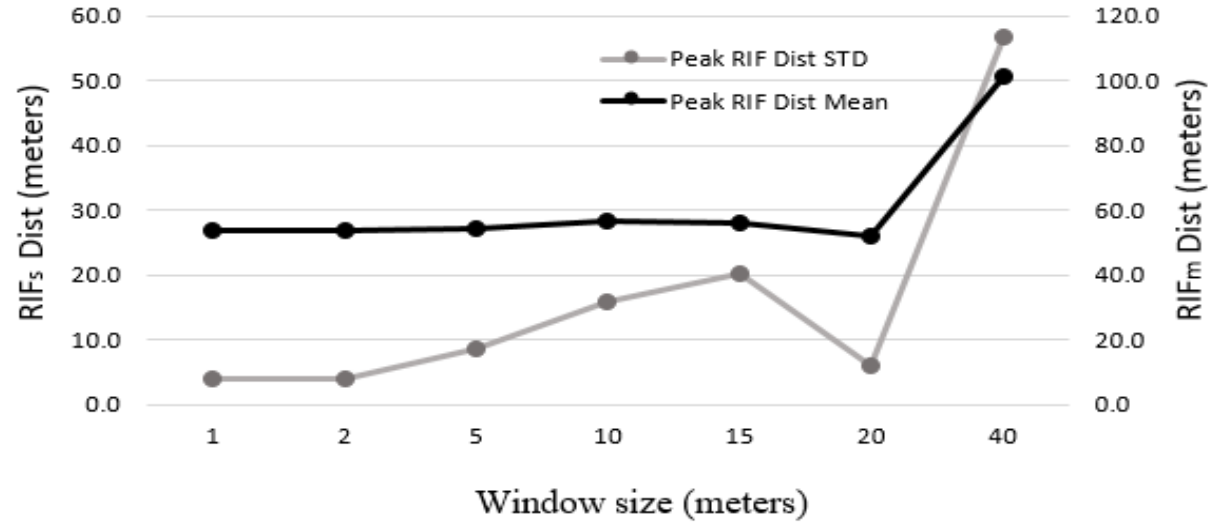

Fig. 5. a) The mean and STD of peak RIFs of varying window size. b) mean of peak RIF distance relative to STD of peak RIF distance at different window size.

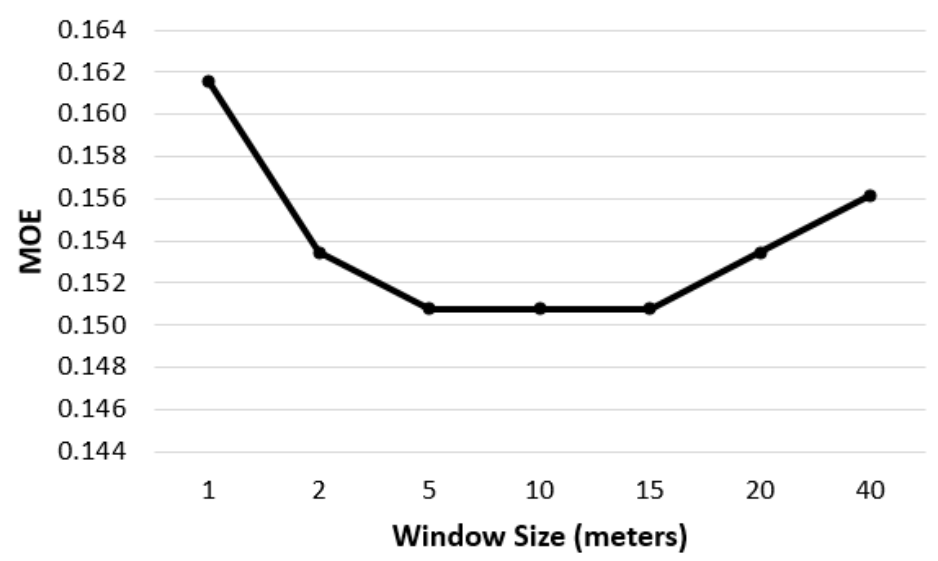

Fig. 6. Margin-of-error of peak RIFs of different window sizes. 
a)

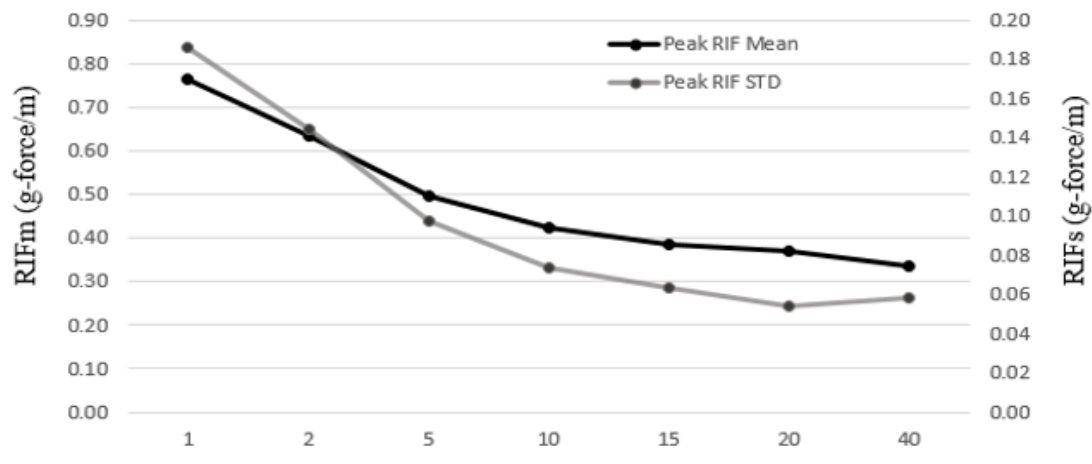

b)

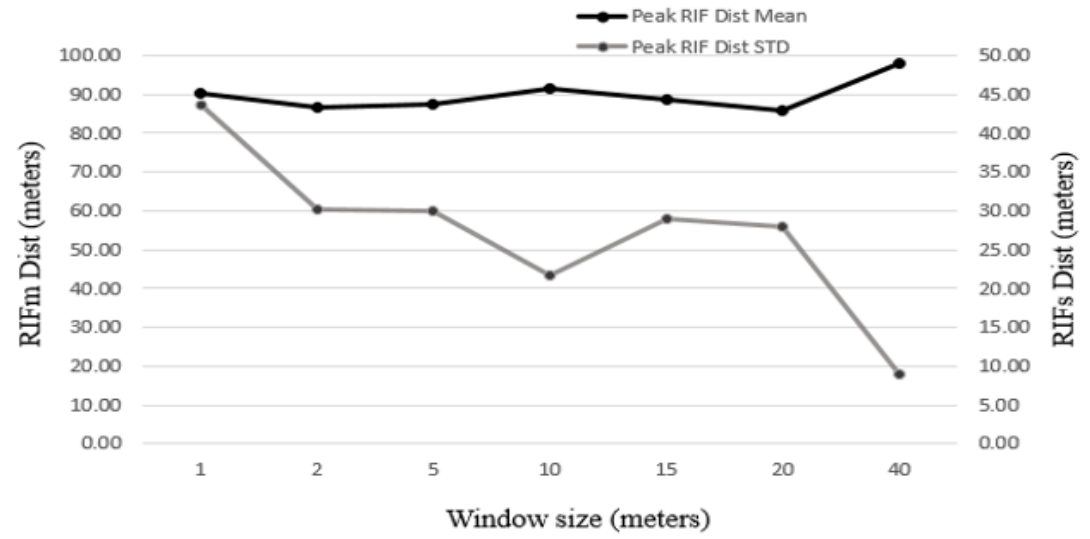

Fig. 7. a) The mean and STD of peak RIFs of varying window size using rail data. b) mean of peak RIF distance relative to STD of peak RIF distance at different window size using rail data. 